\title{
On $r$-dynamic coloring of comb graphs
}

\author{
K. Kalaiselvi ${ }^{1}$, N. Mohanapriya ${ }^{2}$ and J. Vernold Vivin ${ }^{3}$ \\ ${ }^{1}$ Department of Mathematics, Dr. Mahalingam College of Engineering and Technology \\ Pollachi-642 003, Tamil Nadu, India \\ e-mail: kalaiselvi18577@gmail.com \\ ${ }^{2}$ PG and Department of Mathematics, Kongunadu Arts and Science College \\ Coimbatore-641 029, Tamil Nadu, India \\ e-mail: n.mohanamaths@gmail.com \\ ${ }^{3}$ Department of Mathematics, University College of Engineering Nagercoil \\ (A Constituent College of Anna University, Chennai) \\ Konam, Nagercoil-629 004, Tamil Nadu, India \\ e-mail: vernoldvivineyahoo.in
}

Abstract: An $r$-dynamic coloring of a graph $G$ is a proper coloring of $G$ such that every vertex in $V(G)$ has neighbors in at least $\min \{d(v), r\}$ different color classes. The $r$-dynamic chromatic number of graph $G$ denoted as $\chi_{r}(G)$, is the least $k$ such that $G$ has a coloring. In this paper we obtain the $r$-dynamic chromatic number of the central graph, middle graph, total graph, line graph, para-line graph and sub-division graph of the comb graph $P_{n} \odot K_{1}$ denoted by $C\left(P_{n} \odot K_{1}\right)$, $M\left(P_{n} \odot K_{1}\right), T\left(P_{n} \odot K_{1}\right), L\left(P_{n} \odot K_{1}\right), P\left(P_{n} \odot K_{1}\right)$ and $S\left(P_{n} \odot K_{1}\right)$ respectively by finding the upper bound and lower bound for the $r$-dynamic chromatic number of the Comb graph.

Keywords: $r$-dynamic coloring, Comb graph, Central graph, Middle graph, Total graph, Line graph, Sub-division graph, Para-line graph.

2020 Mathematics Subject Classification: 05C15, $05 \mathrm{C} 75$.

\section{Introduction}

In this paper, all graphs are simple and finite. For a graph $G$, let $\delta(G)$ and $\Delta(G)$ denote the minimum and maximum degree of $G$. The $r$-dynamic coloring was first introduced by Montgomery [10]. An $r$-dynamic coloring of a graph is a map $c$ from $V(G)$ to the set of colors such that: 
(i) if $u v \in E(G)$, then $c(u) \neq c(v)$, and

(ii) for each vertex $v \in V(G),|c(N(v))| \geq \min \{d(v), r\}$,

where $N(v)$ denotes the set of all vertices adjacent to $v$ and $d(v)$ its degree and $r$ is a positive integer. The first condition characterizes proper coloring and it is called the adjacency condition and second condition is the $r$-adjacency condition. The $r$-dynamic chromatic number of a graph $G$ is denoted by $\chi_{r}(G)$, is the minimum $k$ such that $G$ admits such a proper $k$-coloring. The 1-dynamic chromatic number of a graph $G$ is equal to its chromatic number. The 2-dynamic chromatic number of a graph $G$ is studied by the name dynamic chromatic number in $[1-4,7]$.

There are many upper bounds and lower bounds for $\chi_{d}(G)$ in terms of graph parameters. For a graph $G$ with $\Delta(G) \geq 3$, Lai et al. [7] proved that $\chi_{d}(G) \leq \Delta(G)+1$, except for a cycle graph $C_{5}$. An upper bound for the dynamic chromatic number of a regular graph $G$ and the independence number of the graph $G, \alpha(G)$, was introduced in [5]. In fact, it was proved that $\chi_{2}(G) \leq \chi(G)+2 \log _{2} \alpha(G)+\mathcal{O}(1)$. Taherkhani gave [11] an upper bound for $\chi_{2}(G)$ in terms of the chromatic number, the maximum degree $\Delta$ and the minimum degree $\delta$ that is $\chi_{2}(G)-\chi(G) \leq\left[\Delta e / \delta \log \left(2 e\left(\Delta^{2}+1\right)\right)\right]$, where $G$ is again a $d$-regular graph. Li et al. proved in [8] that in determining the value of $\chi_{r}(G)$ for planar bipartite graphs with maximum degree at most 3 and arbitrary high girth is an NP-hard problem. Furthermore, Li and Zhou [8] showed that to determine whether there exist a 3-dynamic coloring or not, for a claw free graph with the maximum degree 3 is an NP-complete problem.

\section{Preliminaries}

Let $G$ be a simple and finite graph with vertex $V(G)$ and edge set $E(G)$. The middle graph [9] of $G$ denoted by $M(G)$, is defined as follows, the vertex set of $M(G)$ is $V(G) \cup E(G)$. Two vertices $x, y$ of $M(G)$ are adjacent in $M(G)$ in case one of the following holds: (i) $x, y$ are in $E(G)$ and $x, y$ are adjacent in $G$. (ii) $x$ is in $V(G), y$ is in $E(G)$, and $x, y$ are incident in $G$.

Let $G$ be a graph with vertex set $V(G)$ and edge set $E(G)$. The total graph [9] of $G$, denoted by $T(G)$, is defined in the following way. The vertex set of $T(G)$ is $V(G) \cup E(G)$. Two vertices $x, y$ of $T(G)$ are adjacent in $T(G)$ in case one of the following holds: (i) $x, y$ are in $V(G)$ and $x$ is adjacent to $y$ in $G$. (ii) $x, y$ are in $E(G)$ and $x, y$ are adjacent in $G$. (iii) $x$ is in $V(G), y$ is in $E(G)$, and $x, y$ are incident in $G$.

The central graph [12] $C(G)$ of a graph $G$ is obtained from $G$ by adding an extra vertex on each edge of $G$, and then joining each pair of vertices of the original graph which were previously non-adjacent.

The line graph [6] of $G$, denoted by $L(G)$, is the graph whose vertex set is the edge set of $G$. Two vertices of $L(G)$ are adjacent whenever the corresponding edges of $G$ are adjacent.

The sub-division graph $S(G)$ is obtained simply by inserting a new vertex for each edge of $G$.

The line graph of a sub-division graph is the para-line graph $P(G)$.

Let $P_{n}$ be a path graph with $n$ vertices and $K_{1}$ be a complete graph with one vertex. The comb graph $P_{n} \odot K_{1}$ is defined as the corona product of path graph $P_{n}$ with the complete graph $K_{1}$ by taking one copy of $P_{n}$ and $\left|V\left(P_{n}\right)\right|$ copies of $K_{1}$ and making the $i^{\text {th }}$ vertex of $P_{n}$ adjacent to the $i^{\text {th }}$ copy of $K_{1}$ where $1 \leq i \leq n$. Comb graph has $2 n$ vertices and $2 n-1$ edges. 


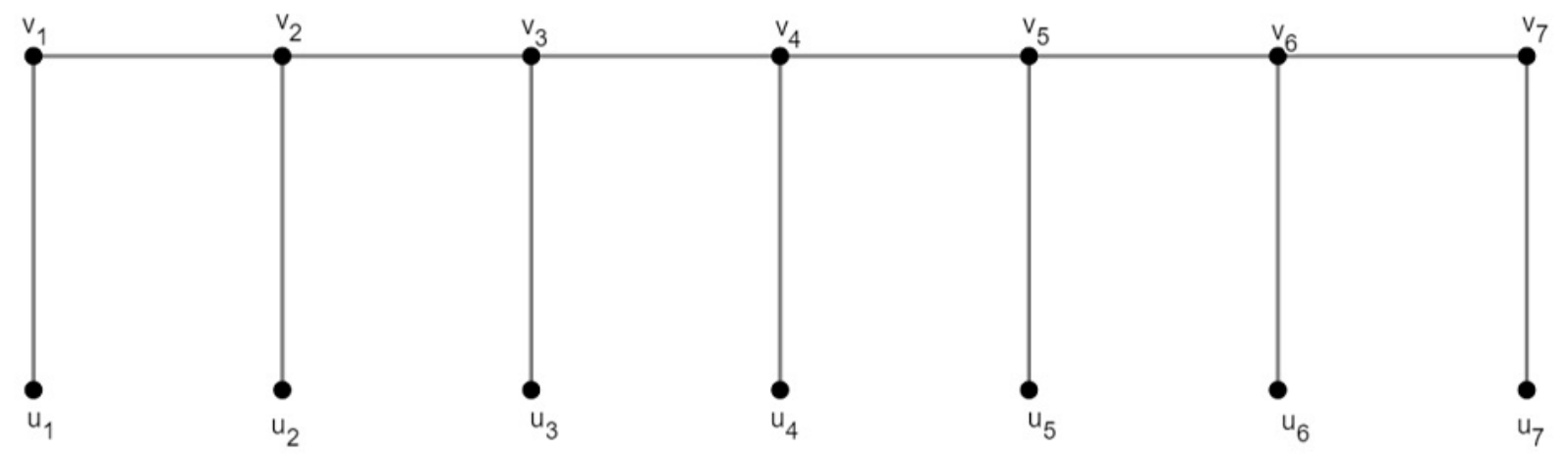

Figure 1. Comb graph $P_{7} \odot K_{1}$

In the following section we obtain the $r$-dynamic chromatic number of the central graph, middle graph, total graph, line graph, para-line graph and sub-division graph of the comb graph $P_{n} \odot K_{1}$ denoted by $C\left(P_{n} \odot K_{1}\right), M\left(P_{n} \odot K_{1}\right), T\left(P_{n} \odot K_{1}\right), L\left(P_{n} \odot K_{1}\right), P\left(P_{n} \odot K_{1}\right)$ and $S\left(P_{n} \odot K_{1}\right)$, respectively.

\section{Main results}

Lemma 3.1. $\chi_{r}(G) \geq \min \{r, \Delta(G)\}+1$.

Theorem 3.2. Let $n \geq 3, C\left(P_{n} \odot K_{1}\right)$ be the central graph of comb graph then

$$
\chi_{r}\left(C\left(P_{n} \odot K_{1}\right)\right)= \begin{cases}n, & \text { for } r=1 \\ 2 n, & \text { for } 2 \leq r \leq \Delta-1 \\ 2 n+3, & \text { for } r=\Delta\end{cases}
$$

Proof. Let

$V\left(C\left(P_{n} \odot K_{1}\right)\right)=\left\{v_{i}: 1 \leq i \leq n\right\} \cup\left\{v_{i}^{\prime}: 1 \leq i \leq n-1\right\} \cup\left\{u_{i}: 1 \leq i \leq n\right\} \cup\left\{u_{i}^{\prime}: 1 \leq i \leq n\right\}$

where $v_{i}^{\prime}$ and $u_{i}^{\prime}$ are the vertices corresponding to the edge $v_{i} v_{i+1}$ and $v_{i} u_{i}$ of $\left(P_{n} \odot K_{1}\right)$, $1 \leq i \leq n$. The maximum and minimum degrees of $C\left(P_{n} \odot K_{1}\right)$ are $\Delta\left(C\left(P_{n} \odot K_{1}\right)\right)=2 n-1$ and $\delta\left(C\left(P_{n} \odot K_{1}\right)\right)=2$. Define the mapping $c: V \rightarrow Z^{+}$.

Case 1: When $r=1$, the $r$-dynamic coloring is $n$ are as follows:

- $c\left(v_{i}\right)=i, 1 \leq i \leq n$

- $c\left(u_{i}\right)=i, 1 \leq i \leq n$

- $c\left(v_{i}^{\prime}\right)= \begin{cases}4, & \text { for } i \neq 3,4,1 \leq i \leq n-1 \\ 2, & \text { for } i=3,4,1 \leq i \leq n-1\end{cases}$

- $c\left(u_{i}^{\prime}\right)= \begin{cases}3, & \text { for } i \neq 3,1 \leq i \leq n \\ 4, & \text { for } i=3,1 \leq i \leq n\end{cases}$

Hence the $r$-adjacency condition is fulfilled, therefore $\chi_{r}\left(C\left(P_{n} \odot K_{1}\right)\right)=n$ for $r=1$.

If $\chi_{r}\left(C\left(P_{n} \odot K_{1}\right)\right)<n$, then the $r$-adjacency condition will not be fulfilled. 
Case 2: When $2 \leq r \leq \Delta-1$, the $r$-dynamic coloring is $2 n$ are as follows:

- $c\left(v_{i}\right)=i, 1 \leq i \leq n$

- $c\left(u_{i}\right)=i+n, 1 \leq i \leq n$

- $c\left(v_{i}^{\prime}\right)=i+n, 1 \leq i \leq n$

- $c\left(u_{i}^{\prime}\right)= \begin{cases}n, & \text { for } i=1,1 \leq i \leq n-1 \\ i-1, & \text { for } 2 \leq i \leq n-1\end{cases}$

Hence the $r$-adjacency condition is fulfilled, therefore $\chi_{r}\left(C\left(P_{n} \odot K_{1}\right)\right)=2 n$ for $2 \leq r \leq \Delta-1$. If $\chi_{r}\left(C\left(P_{n} \odot K_{1}\right)\right)<2 n$, then the $r$-adjacency condition will not be fulfilled.

Case: 3 When $r=\Delta$, the $r$-dynamic coloring is $2 n+3$ are as follows:

- $c\left(v_{i}\right)=i, 1 \leq i \leq n$

- $c\left(u_{i}\right)=i+n, 1 \leq i \leq n$

- $c\left(u_{i}^{\prime}\right)=2 n+1,1 \leq i \leq n$

- $c\left(v_{i}^{\prime}\right)= \begin{cases}2 n+2, & \text { for } i \text { odd } \\ 2 n+3, & \text { for } i \text { even }\end{cases}$

Hence the $r$-adjacency condition is fulfilled, therefore $\chi_{r}\left(C\left(P_{n} \odot K_{1}\right)\right)=2 n+3$ for $r=\Delta$. If $\chi_{r}\left(C\left(P_{n} \odot K_{1}\right)\right)<2 n+3$, then the $r$-adjacency condition will not be fulfilled.

Lemma 3.3. Let $n \geq 4, M\left(P_{n} \odot K_{1}\right)$ be the middle graph of comb graph then

$$
\chi_{r}\left(M\left(P_{n} \odot K_{1}\right)\right) \geq \begin{cases}4, & \text { for } 1 \leq r \leq 3 \\ r+1, & \text { for } 4 \leq r \leq \Delta\end{cases}
$$

Proof. Let

$V\left(M\left(P_{n} \odot K_{1}\right)\right)=\left\{v_{i}: 1 \leq i \leq n\right\} \cup\left\{v_{i}^{\prime}: 1 \leq i \leq n-1\right\} \cup\left\{u_{i}: 1 \leq i \leq n\right\} \cup\left\{u_{i}^{\prime}: 1 \leq i \leq n\right\}$

where $v_{i}^{\prime}$ and $u_{i}^{\prime}$ are the vertices corresponding to the edge $v_{i} v_{i+1}$ and $v_{i} u_{i}$ of $\left(P_{n} \odot K_{1}\right), 1 \leq i \leq n$. By the definition of middle graph the vertices $\left\{v_{i}^{\prime}, v_{i+1}^{\prime}, u_{i+1}^{\prime}, v_{i+1}\right\}$ induces a clique of order 4 , hence $\chi_{r}\left(M\left(P_{n} \odot K_{1}\right)\right) \geq 4$. For $4 \leq r \leq \Delta$ by Lemma $3.1 \chi_{r}(G) \geq \min \{r, \Delta(G)\}+1$. This concludes the proof.

Theorem 3.4. Let $n \geq 4, M\left(P_{n} \odot K_{1}\right)$, then the $r$-dynamic chromatic number of the middle graph of a comb graph is

$$
\chi_{r}\left[M\left(P_{n} \odot K_{1}\right)\right]=\left\{\begin{array}{ll}
4, & \text { for } 1 \leq r \leq 3 \\
r+1, & \text { for } 4 \leq r \leq \Delta
\end{array} .\right.
$$

Proof. The maximum and minimum degrees of $M\left(P_{n} \odot K_{1}\right)$ are $\Delta\left(M\left(\left(P_{n} \odot K_{1}\right)\right)\right)=6$ and $\delta\left(M\left(P_{n} \odot K_{1}\right)\right)=2$. Define the mapping $c: V \rightarrow Z^{+}$. We divide the proof into two cases. 
Case 1: When $1 \leq r \leq 3$, by Lemma 3.3 the lower bound is $\chi_{r}\left(M\left(\left(P_{n} \odot K_{1}\right)\right)\right) \geq 4$. To show the upper bound, we use the following colorings:

- $c\left(v_{1}, v_{2}, \ldots, v_{n}\right)=\{3,4,3,4, \ldots\}$

- $c\left(u_{1}^{\prime}, u_{2}^{\prime}, \ldots, u_{n}^{\prime}\right)=\{4,3,4,3, \ldots\}$

- $c\left(u_{1}, u_{2}, \ldots, u_{n}\right)=1$

- $c\left(v_{1}^{\prime}, v_{2}^{\prime}, \ldots, v_{n}^{\prime}\right)=\{2,1,2,1, \ldots\}$

Thus we require 4 colors, that is $\chi_{r}\left(M\left(P_{n} \odot K_{1}\right)\right) \geq 4$. Hence $\chi_{r}\left(M\left(P_{n} \odot K_{1}\right)\right)=4$.

Case 2: When $4 \leq r \leq \Delta$, by Lemma 3.3 the lower bound is $\chi_{r}\left(M\left(\left(P_{n} \odot K_{1}\right)\right)\right) \geq 4$. To show the upper bound, we use the following colorings.

Subcase (i): when $r=4$, consider the coloring:

$$
\begin{aligned}
& -c\left(v_{1}, v_{2}, \ldots, v_{n}\right)=\{1,2,3,1,2,3, \ldots\} \\
& -c\left(v_{1}^{\prime}, v_{2}^{\prime}, \ldots, v_{n}^{\prime}\right)=\{3,1,2,3,1,2, \ldots\} \\
& \text { - } c\left(u_{1}^{\prime}, u_{2}^{\prime}, \ldots, u_{n}^{\prime}\right)=\{r, r+1, r, r+1, \ldots\} \\
& \text { - } c\left(u_{1}, u_{2}, \ldots, u_{n}\right)=\{r+1, r, r+1, r, \ldots\}
\end{aligned}
$$

Thus we require $r+1$ colors when $r=4$.

Subcase (ii): When $r=5$, consider the coloring:

- $c\left(v_{1}, v_{2}, \ldots, v_{n}\right)=\{2,3,2,3, \ldots\}$

- $c\left(v_{1}^{\prime}, v_{2}^{\prime}, \ldots, v_{n}^{\prime}\right)=\{1,4,1,4, \ldots\}$

- $c\left(u_{1}^{\prime}, u_{2}^{\prime}, \ldots, u_{n}^{\prime}\right)=\{r, r+1, r, r+1, \ldots\}$

- $c\left(u_{1}, u_{2}, \ldots, u_{n}\right)=\{r+1, r, r+1, r, \ldots\}$

Thus we require $r+1$ colors when $r=5$.

Subcase (iii): When $r=6$, consider the coloring:

$$
\begin{aligned}
& -c\left(v_{1}, v_{2}, \ldots, v_{n}\right)=\{4,1,5,2,3,4,1,5,2,3, \ldots\} \\
& -c\left(v_{1}^{\prime}, v_{2}^{\prime}, \ldots, v_{n}^{\prime}\right)=\{2,3,4,1,5,2,3,4,1,5, \ldots\} \\
& \text { - } c\left(u_{1}^{\prime}, u_{2}^{\prime}, \ldots, u_{n}^{\prime}\right)=\{r, r+1, r, r+1, \ldots\} \\
& \text { - } c\left(u_{1}, u_{2}, \ldots, u_{n}\right)=\{r+1, r, r+1, r, \ldots\}
\end{aligned}
$$

Thus we require $r+1$ colors when $r=6$.

Now from the subcases (i), (ii) and (iii) the $r$-adjacency condition is fulfilled. Therefore, $\chi_{r}\left(M\left(P_{n} \odot K_{1}\right)\right) \leq r+1$. Hence $\chi_{r}\left(M\left(P_{n} \odot K_{1}\right)\right)=r+1$.

Lemma 3.5. Let $n \geq 3, T\left(P_{n} \odot K_{1}\right)$ be the total graph of comb graph, then

$$
\chi_{r}\left(T\left(P_{n} \odot K_{1}\right)\right) \geq\left\{\begin{array}{l}
4, \quad \text { for } 1 \leq r \leq 3 \\
r+1, \quad \text { for } 4 \leq r \leq \Delta
\end{array} .\right.
$$

Proof. Let

$V\left(T\left(P_{n} \odot K_{1}\right)\right)=\left\{v_{i}: 1 \leq i \leq n\right\} \cup\left\{v_{i}^{\prime}: 1 \leq i \leq n-1\right\} \cup\left\{u_{i}: 1 \leq i \leq n\right\} \cup\left\{u_{i}^{\prime}: 1 \leq i \leq n\right\}$, where $v_{i}^{\prime}$ and $u_{i}^{\prime}$ are the vertices corresponding to the edge $v_{i} v_{i+1}$ and $v_{i} u_{i}$ of $\left(P_{n} \odot K_{1}\right), 1 \leq i \leq n$.

By the definition of total graph the vertices $\left\{v_{i}^{\prime}, v_{i+1}^{\prime}, u_{i+1}^{\prime}, v_{i+1}\right\}$ induce a clique of order 4 , hence $\chi_{r}\left(T\left(P_{n} \odot K_{1}\right)\right) \geq 4$. For $4 \leq r \leq \Delta$ by Lemma $3.1 \chi_{r}(G) \geq \min \{r, \Delta(G)\}+1$. This concludes the proof. 
Theorem 3.6. Let $n \geq 3, T\left(P_{n} \odot K_{1}\right)$ the $r$-dynamic chromatic number of the total graph of comb graph is

$$
\chi_{r}\left(T\left(P_{n} \odot K_{1}\right)\right)= \begin{cases}4, & \text { for } 1 \leq r \leq 3 \\ r+1, & \text { for } 4 \leq r \leq \Delta\end{cases}
$$

Proof. The maximum and minimum degrees of $T\left(P_{n} \odot K_{1}\right)$ are $\Delta\left(T\left(P_{n} \odot K_{1}\right)\right)=6$ and $\delta\left(T\left(P_{n} \odot K_{1}\right)\right)=2$. We divide the proof into two cases. Define the mapping $c: V \rightarrow Z^{+}$.

Case 1: When $1 \leq r \leq 3$, by Lemma 3.5 the lower bound is $\chi_{r}\left(T\left(P_{n} \odot K_{1}\right)\right) \geq 4$. To show the upper bound, we use the following colorings:

- $c\left(v_{1}, v_{2}, \ldots, v_{n}\right)=\{2,3,2,3, \ldots\}$

- $c\left(u_{1}^{\prime}, u_{2}^{\prime}, \ldots, u_{n}^{\prime}\right)=\{3,2,3, \ldots\}$

- $c\left(u_{1}, u_{2}, \ldots, u_{n}\right)=\{1,4,1,4, \ldots\}$

- $c\left(v_{1}^{\prime}, v_{2}^{\prime}, \ldots, v_{n}^{\prime}\right)=\{4,1,4,1, \ldots\}$

Thus we require 4 colors that is $\chi_{r}\left(T\left(P_{n} \odot K_{1}\right)\right) \leq 4$. Hence $\chi_{r}\left(T\left(P_{n} \odot K_{1}\right)\right)=4$.

Case 2: When $4 \leq r \leq \Delta$, by Lemma 3.5 the lower bound is $\chi_{r}\left(T\left(P_{n} \odot K_{1}\right)\right) \geq 4$. To show the upper bound, we use the following colorings:

Subcase (i): When $r=4$, consider the coloring:

$$
\begin{aligned}
& -c\left(v_{1}, v_{2}, \ldots, v_{n}\right)=\{1,2,3,1,2,3, \ldots\} \\
& -c\left(v_{1}^{\prime}, v_{2}^{\prime}, \ldots, v_{n}^{\prime}\right)=\{3,1,2,3,1,2, \ldots\} \\
& -c\left(u_{1}^{\prime}, u_{2}^{\prime}, \ldots, u_{n}^{\prime}\right)=\{r, r+1, r, r+1, \ldots\} \\
& -c\left(u_{1}, u_{2}, \ldots, u_{n}\right)=\{r+1, r, r+1, r, \ldots\}
\end{aligned}
$$

Thus we require $r+1$ colors when $r=4$.

Subcase (ii): When $r=5$, consider the coloring:

$$
\begin{aligned}
& -c\left(v_{1}, v_{2}, \ldots, v_{n}\right)=\{2,3,2,3, \ldots\} \\
& -c\left(v_{1}^{\prime}, v_{2}^{\prime}, \ldots, v_{n}^{\prime}\right)=\{1,4,1,4, \ldots\} \\
& -c\left(u_{1}^{\prime}, u_{2}^{\prime}, \ldots, u_{n}^{\prime}\right)=\{r, r+1, r, r+1, \ldots\} \\
& -c\left(u_{1}, u_{2}, \ldots, u_{n}\right)=\{r+1, r, r+1, r, \ldots\}
\end{aligned}
$$

Thus we require $r+1$ colors when $r=5$.

Subcase (iii): When $r=6$, consider the coloring:

$$
\begin{aligned}
& -c\left(v_{1}, v_{2}, \ldots, v_{n}\right)=\{4,1,5,2,3,4,1,5,2,3, \ldots\} \\
& -c\left(v_{1}^{\prime}, v_{2}^{\prime}, \ldots, v_{n}^{\prime}\right)=\{2,3,4,1,5,2,3,4,1,5, \ldots\} \\
& -c\left(u_{1}^{\prime}, u_{2}^{\prime}, \ldots, u_{n}^{\prime}\right)=\{r, r+1, r, r+1, \ldots\} \\
& -c\left(u_{1}, u_{2}, \ldots, u_{n}\right)=\{r+1, r, r+1, r, \ldots\}
\end{aligned}
$$

Thus we require $r+1$ colors when $r=6$.

Now from the subcases (i), (ii) and (iii) the $r$-adjacency condition fulfilled. Therefore, $\chi_{r}\left(T\left(P_{n} \odot K_{1}\right)\right) \leq r+1$. Hence $\chi_{r}\left(T\left(P_{n} \odot K_{1}\right)\right)=r+1$. 
Lemma 3.7. Let $n \geq 4, L\left(P_{n} \odot K_{1}\right)$ be the line graph of comb graph, then

$$
\chi_{r}\left(L\left(P_{n} \odot K_{1}\right)\right) \geq\left\{\begin{array}{l}
3, \text { for } 1 \leq r \leq 2 \\
4, \text { for } r=3 \\
5, \text { for } r=4
\end{array} .\right.
$$

Proof. Let $V\left(L\left(P_{n} \odot K_{1}\right)\right)=e_{1}, e_{2}, e_{3}, \ldots, e_{n}, e_{1}^{\prime}, e_{2}^{\prime}, e_{3}^{\prime}, \ldots, e_{n-1}^{\prime}$. The maximum and minimum degrees of $L\left(P_{n} \odot K_{1}\right)$ are $\Delta\left(L\left(P_{n} \odot K_{1}\right)\right)=4$ and $\delta\left(L\left(P_{n} \odot K_{1}\right)\right)=1$. For $1 \leq r \leq 2$ by the definition of line graph the vertices $e_{i}, e_{i}^{\prime}, e_{i+1}^{\prime}$ induces a clique of order 3 . Hence $\chi_{r}\left(L\left(P_{n} \odot K_{1}\right)\right) \geq 3$.

For $r=3$ by Lemma 3.1

$\chi_{r}\left(L\left(P_{n} \odot K_{1}\right)\right) \geq \min \left\{r, \Delta\left(L\left(P_{n} \odot K_{1}\right)\right\} \geq \min \left\{3, \Delta\left(L\left(P_{n} \odot K_{1}\right)\right)\right\}+1=3+1=4\right.$.

For $r=4$ by Lemma 3.1

$\chi_{r}\left(L\left(P_{n} \odot K_{1}\right)\right) \geq \min \left\{r, \Delta\left(L\left(P_{n} \odot K_{1}\right)\right)\right\} \geq \min \left\{4, \Delta\left(L\left(P_{n} \odot K_{1}\right)\right)\right\}+1=4+1=5$.

This concludes the proof.

Theorem 3.8. Let $n \geq 4, L\left(P_{n} \odot K_{1}\right)$ the r-dynamic chromatic number of a line graph of comb graph is

$$
\chi_{r}\left(L\left(P_{n} \odot K_{1}\right)\right)=\left\{\begin{array}{l}
3, \text { for } 1 \leq r \leq 2 \\
4, \text { for } r=3 \\
5, \text { for } r=4
\end{array} .\right.
$$

Proof. Define the mapping $c: V \rightarrow Z^{+}$. We divide the proof into three cases.

Case 1: For $1 \leq r \leq 2$. By the Lemma 3.7 the lower bound is $\chi_{r}\left(L\left(P_{n} \odot K_{1}\right)\right) \geq 3$. To show the upper bound, we use the following colorings:

- $c\left(e_{1}, e_{2}, \ldots, e_{n}\right)=1$

- $c\left(e_{1}^{\prime}, e_{2}^{\prime}, \ldots, e_{n}^{\prime}\right)=\{2,3,2,3, \ldots\}$

Thus we require 3 colors, that is $\chi_{r}\left(L\left(P_{n} \odot K_{1}\right)\right) \leq 3$. Hence $\chi_{r}\left(L\left(P_{n} \odot K_{1}\right)\right)=3$ when $1 \leq r \leq 2$.

Case 2: For $r=3$. By the Lemma 3.7 the lower bound is $\chi_{r}\left(L\left(P_{n} \odot K_{1}\right)\right) \geq 4$. The $r$-dynamic coloring are as follows:

- $c\left(e_{1}, e_{2}, \ldots, e_{n}\right)=\{1,3,1,3, \ldots\}$

- $c\left(e_{1}^{\prime}, e_{2}^{\prime}, \ldots, e_{n}^{\prime}\right)=\{2,4,2,4, \ldots\}$

Thus we require 4 colors, that is $\chi_{r}\left(L\left(P_{n} \odot K_{1}\right)\right) \leq 4$. Hence $\chi_{r}\left(L\left(P_{n} \odot K_{1}\right)\right)=4$ when $r=3$.

Case 3: For $r=4$. By the Lemma 3.7 the lower bound is $\chi_{r}\left(L\left(P_{n} \odot K_{1}\right)\right) \geq 5$. The $r$-dynamic coloring are as follows:

- $c\left(e_{1}, e_{2}, \ldots, e_{n}\right)=\{1,3,1,3, \ldots\}$

- $c\left(e_{1}^{\prime}, e_{2}^{\prime}, \ldots, e_{n}^{\prime}\right)=\{2,4,5,2,4,5, \ldots\}$

Thus we require 5 colors, that is $\chi_{r}\left(L\left(P_{n} \odot K_{1}\right)\right) \leq 5$. Hence $\chi_{r}\left(L\left(P_{n} \odot K_{1}\right)\right)=5$ when $r=4$. 
Lemma 3.9. Let $n \geq 3, P\left(P_{n} \odot K_{1}\right)$ be the para-line graph of comb graph then

$$
\chi_{r}\left(P\left(P_{n} \odot K_{1}\right)\right) \geq\left\{\begin{array}{l}
3, \text { for } 1 \leq r \leq 2 \\
4, \text { for } r=\Delta
\end{array} .\right.
$$

Proof. Let $V\left(P\left(P_{n} \odot K_{1}\right)\right)=\left\{e_{i} ; 1 \leq i \leq 2 n\right\} \cup\left\{e_{i}^{\prime} ; 1 \leq i \leq 2 n-2\right\}$, where $e_{i}$ is the vertex corresponding to the edge $v_{i} v_{i+1}$ and $e_{i}^{\prime}$ is the vertex corresponding to the edge $v_{i} u_{i}$ of $\left(P_{n} \odot k_{1}\right)$, $1 \leq i \leq n$. For $1 \leq r \leq 2$, by the definition of para-line graph the vertices $e_{2 i+2}, e_{i+1}^{\prime}, e_{i+2}^{\prime}$ induces a clique of order 3 . Hence $\chi_{r}\left(P\left(P_{n} \odot K_{1}\right)\right) \geq 3$.

For $r=\Delta$ by Lemma $3.1 \chi_{r}\left(L\left(P_{n} \odot K_{1}\right)\right) \geq \min \left\{r, \Delta\left(P\left(P_{n} \odot K_{1}\right)\right)\right\}+1=3+1=4$. Therefore $\chi_{r}\left(L\left(P_{n} \odot K_{1}\right)\right) \geq 4$. This concludes the proof.

Theorem 3.10. Let $n \geq 3, P\left(P_{n} \odot K_{1}\right)$ the $r$-dynamic chromatic number of para-line graph of a comb graph is

$$
\chi_{r}\left(P\left(P_{n} \odot K_{1}\right)\right)=\left\{\begin{array}{l}
3, \text { for } 1 \leq r \leq 2 \\
4, \text { for } r=\Delta
\end{array} .\right.
$$

Proof. The maximum and minimum degrees of $L\left(P_{n} \odot K_{1}\right)$ are $\Delta\left(L\left(P_{n} \odot K_{1}\right)\right)=3$ and $\delta\left(L\left(P_{n} \odot K_{1}\right)\right)=1$. Define the mapping $c: V \rightarrow Z^{+}$.

Case 1: For $1 \leq r \leq 2$. By the Lemma 3.9 the lower bound is $\chi_{r}\left(L\left(P_{n} \odot K_{1}\right)\right) \geq 3$. To show the upper bound, we assign colors as follows:

- $c\left(e_{1}, e_{2}, \ldots, e_{n}\right)=\{1,2,1,2, \ldots\}$

- $c\left(e_{1}^{\prime}, e_{2}^{\prime}, \ldots, e_{n}^{\prime}\right)=\{1,3,1,3, \ldots\}$

Thus we require 3 colors, that is $\chi_{r}\left(P\left(P_{n} \odot K_{1}\right)\right) \leq 3$. Hence $\chi_{r}\left(P\left(P_{n} \odot K_{1}\right)\right)=3$ when $1 \leq r \leq 2$.

Case 2: For $r=3$. By the Lemma 3.9 the lower bound is $\chi_{r}\left(P\left(P_{n} \odot K_{1}\right)\right) \geq 4$. To show the upper bound, we assign colors as follows:

- $c\left(e_{1}, e_{2}, \ldots, e_{2 n-1}\right)=\{3,1,3,1, \ldots\}$ and $c\left(e_{2 n}\right)=4$

- $c\left(e_{1}^{\prime}, e_{2}^{\prime}, \ldots, e_{n}^{\prime}\right)=\{1,2,1,2, \ldots\}$

Thus we require 4 colors, that is $\chi_{r}\left(P\left(P_{n} \odot K_{1}\right)\right) \leq 4$. Hence $\chi_{r}\left(P\left(P_{n} \odot K_{1}\right)\right)=4$ when $r=3$.

Theorem 3.11. Let $n \geq 3$, and $S\left(P_{n} \odot K_{1}\right)$ be the sub-division graph of $\left(P_{n} \odot K_{1}\right)$, then

$$
\chi_{r}\left(S\left(P_{n} \odot K_{1}\right)\right)=r+1,1 \leq r \leq 3
$$

Proof. Let $V\left(S\left(P_{n} \odot K_{1}\right)\right)=\left\{v_{i}: 1 \leq i \leq n\right\} \cup\left\{v_{i}^{\prime}: 1 \leq i \leq n-1\right\} \cup\left\{u_{i}: 1 \leq i \leq n\right\} \cup$ $\left\{u_{i}^{\prime}: 1 \leq i \leq n\right\}$ where $v_{i}^{\prime}$ and $u_{i}^{\prime}$ are the vertex corresponding to the edge $v_{i} v_{i+1}$ and $v_{i} u_{i}$ of $\left(P_{n} \odot K_{1}\right), 1 \leq i \leq n$. The maximum and minimum degrees of $S\left(P_{n} \odot K_{1}\right)$ are $\Delta\left(S\left(P_{n} \odot K_{1}\right)\right)=$ 3 and $\delta\left(S\left(P_{n} \odot K_{1}\right)\right)=1$. By Lemma $3.1 \chi_{r}\left(S\left(P_{n} \odot K_{1}\right)\right) \geq \min \left\{r, \Delta\left(S\left(P_{n} \odot K_{1}\right)\right)\right\}+1=r+1$, for $1 \leq r \leq 3$. To show the upper bound, we assign colors as follows: 
Case 1: When $r=1$,

- $c\left(v_{1}, v_{2}, \ldots, v_{n}\right)=1$

- $c\left(v_{1}^{\prime}, v_{2}^{\prime}, \ldots, v_{n}^{\prime}\right)=2$

- $c\left(u_{1}^{\prime}, u_{2}^{\prime}, \ldots, u_{n}^{\prime}\right)=2$

- $c\left(u_{1}, u_{2}, \ldots, u_{n}\right)=1$

Thus we require 2 colors, that is $\chi_{r}\left(S\left(P_{n} \odot K_{1}\right)\right) \leq 2$. Hence $\chi_{r}\left(S\left(P_{n} \odot K_{1}\right)\right)=2$ when $r=1$.

Case 2: When $r=2$,

- $c\left(v_{1}, v_{2}, \ldots, v_{n}\right)=\{1,2,1,2, \ldots\}$

- $c\left(v_{1}^{\prime}, v_{2}^{\prime}, \ldots, v_{n}^{\prime}\right)=3$

- $c\left(u_{1}^{\prime}, u_{2}^{\prime}, \ldots, u_{n}^{\prime}\right)=\{2,1,2,1, \ldots\}$

- $c\left(u_{1}, u_{2}, \ldots, u_{n}\right)=3$

Thus we require 3 colors, that is $\chi_{r}\left(S\left(P_{n} \odot K_{1}\right)\right) \leq 3$. Hence $\chi_{r}\left(S\left(P_{n} \odot K_{1}\right)\right)=3$ when $r=2$.

Case 3: When $r=3$,

- $c\left(v_{1}, v_{2}, \ldots, v_{n}\right)=\{1,2,1,2, \ldots\}$

- $c\left(v_{1}^{\prime}, v_{2}^{\prime}, \ldots, v_{n}^{\prime}\right)=\{3,4,3,4, \ldots\}$

- $c\left(u_{1}^{\prime}, u_{2}^{\prime}, \ldots, u_{n}^{\prime}\right)=\{2,1,2,1, \ldots\}$

- $c\left(u_{1}, u_{2}, \ldots, u_{n}\right)=4$

Thus we require 4 colors, that is $\chi_{r}\left(S\left(P_{n} \odot K_{1}\right)\right) \leq 4$. Hence $\chi_{r}\left(S\left(P_{n} \odot K_{1}\right)\right)=4$ when $r=3$. Therefore $\chi_{r}\left(S\left(P_{n} \odot K_{1}\right)\right) \leq r+1,1 \leq r \leq 3$. Hence $\chi_{r}\left(S\left(P_{n} \odot K_{1}\right)\right)=r+1$, $1 \leq r \leq 3$

\section{Conclusion}

In this paper we have investigated the $r$-dynamic chromatic number of some operations such as central graph, middle graph, total graph, line graph, para-line graph, and subdivision graph of the comb graph $P_{n} \odot K_{1}$. We have used the upper bound and lower bound method to obtain the $r$-dynamic chromatic number. Let $G$ be any connected graph, till now there is no sharp lower bound for the $r$-dynamic chromatic number, so it is left as an open problem.

\section{Acknowledgements}

The authors express their gratitude to the anonymous reviewers for their thorough reading, insightful remarks, and helpful suggestions that improved the content of this manuscript. 


\section{References}

[1] Ahadi, A., Akbari, S., Dehghan, A., \& Ghanbari, M. (2012). On the difference between chromatic number and dynamic chromatic number of graphs. Discrete Mathematics, 312, 2579-2583.

[2] Akbari, S., Ghanbari, M., \& Jahanbekam, S. (2009). On the list dynamic coloring of graphs. Discrete Applied Mathematics, 157, 3005-3007.

[3] Akbari, S., Ghanbari, M., \& Jahanbekam, S. (2010). On the dynamic chromatic number of graphs. AMS Contemporary Mathematics, 531, 11-18.

[4] Alishahi, M. (2012). Dynamic chromatic number of regular graphs. Discrete Applied Mathematics, 160, 2098-2103.

[5] Dehghan, A., \& Ahadi, A. (2012). Upper bounds for the 2-hued chromatic number of graphs in terms of the independence number. Discrete Applied Mathematics, 160(15), 2142-2146.

[6] Harary, F. (1969). Graph Theory, Narosa Publishing home, New Delhi.

[7] Lai, H. J., Montgomery, B., \& Poon, H. (2003). Upper bounds of dynamic chromatic number. Ars Combinatorica, 68, 193-201.

[8] Li, X., \& Zhou, W. (2008). The 2nd-order conditional 3-coloring of claw-free graphs. Theoretical Computer Science, 396, 151-157.

[9] Michalak, D. (1983). On middle and total graphs with coarseness number equal 1. Lecture Notes in Mathematics, 1018, Springer Verlag Graph Theory, Lagow proceedings, Berlin Heidelberg, New York, Tokyo, 139-150.

[10] Montgomery, B. (2001). Dynamic Coloring of Graphs, ProQuest LLC, Ann Arbor, MI, Ph.D. Thesis, West Virginia University.

[11] Taherkhani, A. (2016). $r$-Dynamic chromatic number of graphs. Discrete Applied Mathematics, 201, 222-227.

[12] Vernold Vivin, J. (2007). Harmonious Coloring of Total Graphs, n-leaf, Central Graphs and Circumdetic Graphs, Ph.D. Thesis, Bharathiar University, Coimbatore, India. 